\title{
A propos de l'évolution \\ d'Hymenolepis nana var. fraterna \\ chez des hôtes intermédiaires inhabituels
}

\author{
par N. LEGER et R. CAVIER \\ Laboratoire de Parasitologie ( $P^{r}$ R. CAVIER), \\ Faculté de Pharmacie, 4, avenue de l'Observatoire, F. 75-Paris (VI')
}

\section{Résumé}

Des Insectes normalement non infestables par Hymenolepis nana var. fraterna, peuvent être cependant parasités par l'introduction directe dans leur cavité générale, d'embryons hexacanthes éclos « in vitro ».

D'autre part l'infestation de ces mêmes Insectes par la voie buccale conduit à la libération, dans la lumière du tube digestif, d'embryons hexacanthes viables.

C'est donc au niveau du passage de la paroi intestinale que se situe l'obstacle à l'infestation. Le rôle de la structure de la membrane péritrophique est discuté.

\section{Summary}

Insects, normally non possibly infested by Hymenolepis nana var. fraterna, may nevertheless be infested by introduction in the body cavity of six-hooks embryos hatched « in vitro ».

On the other hand, oral infestation of these same Insects leads to the hatching in the gut of viable six-hooks embryos.

It is then at the crossing of the intestinal wall that is found the obstacle to infection.

The function of the peritrophic membrane structure is discussed. 
Dans un précédent article (1), nous avions exposé un travail relatif aux réactions observées chez des hôtes inhabituels du cysticercoïde d'Hymenolepis nana variété fraterna.

Ces hôtes, des Insectes non infestables par la voie buccale, avaient été parasités par l'introduction directe, dans la cavité générale, d'embryons éclos in vitro.

C'est ainsi que chez Leucophaea maderae, nous avions obtenu des formations particulières : énormes capsules pigmentées groupant un grand nombre de scolex d'aspect plus ou moins anormal.

Or, malgré les anomalies constatées, ces formations, administrées à des souris indemnes de toute contamination antérieure, donnèrent naissance à des formes adultes de morphologie parfaitement normale. Ainsi donc, bien que Leucophaea maderae ne soit pas infestable par la voie buccale, sa cavité générale constitue un milieu propice à l'évolution du parasite jusqu'au stade cysticercoïde infestant pour le Rongeur.

La question était alors de savoir à quel niveau se situait l'obstacle qui s'opposait à la contamination par la voie digestive.

Nous avons tenté l'infestation d'un certain nombre d'Insectes par les deux techniques précédemment décrites :

- technique du repas infestant,

- technique de l'injection intra-cavitaire d'embryons éclos in vitro.

Les insectes essayés se répartissent en trois catégories :

\section{$1^{\circ}$ INSECTES INFESTABLES PAR LA VOIE BUCCALE :}

Nous avons pu réaliser de cette manière l'infestation expérimentale de deux espèces de Grillons: Gryllus domesticus et Gryllus bimaculatus, ainsi que de deux espèces de Criquets: Locusta migratoria et Schistocerca gregaria. Notons qu'avant nous, seul Pujatti (2) avait évoqué le rôle éventuel d'Orthoptères dans la transmission du parasite. Il décrit des cysticercoïdes ressemblant à ceux d'Hymenolepis nana chez $2 \%$ des Gryllodes sigillatus capturés dans la cuisine d'un hôpital militaire aux Indes.

Les cysticercoïdes obtenus chez les Orthoptères expérimentés étaient souvent très abondants et de morphologie normale. Cependant, ils nous ont semblé nettement plus grands que ceux du même âge obtenus chez l'hôte habituel : Tenebrio molitor. La queue en particulier atteignait souvent une taille importante. Nous sommes tentés de croire que le volume atteint par cet appendice est fonction de l'espace dont le parasite dispose, c'est-à-dire de la grandeur de la cavité générale de l'Insecte chez lequel il se développe.

Signalons que nous avons échoué dans nos essais d'infestation de Stegobium paniceum par la voie buccale. La voie intra-cavitaire n'a pas été essayée en raison des difficultés techniques liées à la petite taille de cet insecte. 
$2^{\circ}$ INSECTES NON-INFESTABLES, NI PAR LA VOIE BUCCALE, NI PAR LA VOIE INTRA-CAVITAIRE :

Parmi les espèces essayées seul un Phasme : Carausius morosus (Dictyoptère) s'est montré impropre à assurer le développement du parasite. Infesté par voie intra-cavitaire, il a réagi par la formation de capsules pigmentées autour de débris d'œufs, mais nous n'avons pas retrouvé chez lui de cysticercoïdes.

$3^{\circ}$ INSECtes NON INFESTABles PAR LA VOIE BUCCALE, MAIS INFESTABles PAR LA VoIE INTRA-CAVITAIRE :

Les trois espèces de Blattes que nous avons expérimentées appartiennent à ce groupe: Periplaneta americana, Leucophaea maderae et Blabera fusca.

Rappelons qu'en dépit des réactions observées chez l'hôte et des anomalies morphologiques présentées par le parasite, les cysticercoïdes obtenus dans ces conditions sont infestants pour la Souris.

Dès lors, deux hypothèses sont à envisager :

ou bien, l'œuf ne peut libérer l'embryon hexacanthe au niveau du tube digestif,

ou bien, l'embryon libéré ne peut pas franchir la paroi de l'intestin. Nous avons tenté d'apporter la solution à ce problème.

\section{Etude de la destinée de l'œuf au cours du transit intestinal}

\section{A. - Technique :}

Des blattes (Periplaneta et Leucophaea) ont été nourries avec des proglottis mûrs d'Hymenolepis nana. Chaque insecte a été gavé individuellement, ce qui nous a permis de noter l'heure du repas infestant.

Les animaux mis à l'étuve à $30^{\circ} \mathrm{C}$ ont été ensuite sacrifiés au bout de temps variables et leur contenu intestinal examiné au microscope entre lame et lamelle.

\section{B. - Résultats :}

Le matériel infestant est ingéré avec facilité et la membrane externe de l'œuf est brisée; dès la première demi-heure, on retrouve de nombreuses coques vides.

$\mathrm{Au}$ bout de 1 heure 30 à 2 heures, on observe des embryons hexacanthes libres dans la lumière intestinale.

Le contenu intestinal recueilli à ce moment a été injecté dans la cavité générale de blattes neuves.

Les embryons ainsi transplantés se sont développés normalement et ont donné en quelques jours des cysticercoïdes mûrs.

\section{C. - Conclusions :}

Ces expériences prouvent que le tube digestif de la Blatte constitue un milieu propice à l'éclosion des œufs. 
Puisque, d'autre part, le cysticercoïde se développe normalement dans la cavité générale, c'est au niveau de la paroi intestinale que se situe l'obstacle.

\section{Etude de la structure histologique de l'intestin chez les divers Insectes expérimentés}

Chez l'Insecte, c'est au niveau de l'intestin moyen, non chitineux, que s'effectue l'essentiel de l'absorption digestive et sans doute le passage de la plupart des parasites.

L'intestin moyen est parfois doublé intérieurement par la membrane péritrophique, enveloppe simple ou formée de plusieurs couches, contenant de la chitine et entourant les aliments.

Une grande confusion existe dans la littérature quant à la présence ou à l'absence de cette membrane chez les divers groupes d'Insectes. Il existe deux types de membrane péritrophique :

$1^{\circ}$ la membrane péritrophique de sécrétion produite par un anneau de cellules situé près de la jonction de l'intestin antérieur et de l'intestin moyen,

$2^{\circ}$ la membrane péritrophique de délamination produite par une délamination périodique de la totalité de la surface de l'intestin moyen.

$3^{\circ}$ enfin, certains Insectes possèdent une péritrophique d'origine mixte.

Les espèces sur lesquelles nous avons mené notre expérimentation appartiennent à des ordres chez lesquels, on trouve des membranes péritrophiques du second type.

Nous avons étudié et comparé la structure histologique de l'intestin moyen chez les insectes infestables par la voie buccale et chez ceux qui ne le sont pas.

Les Insectes non infestables par la voie buccale présentent tous une membrane péritrophique nette, souvent importante d'aspect continu. (photos 1 et 2).

$\mathrm{Au}$ contraire, les Insectes infestables par la voie buccale ont une péritrophique peu ou pas visible, d'aspect discontinu (photos 3, 4 et 5). Il semble probable que c'est la structure de la péritrophique, continue ou discontinue, qui conditionne le passage éventuel des embryons hexacanthes de la lumière du tube digestif à la cavité générale. En effet, la membrane péritrophique de délamination se présente comme un réseau de fibrilles de chitine, généralement orientées selon trois directions formant entre elles des angles de $60^{\circ}$, et supportant une substance amorphe.

Dans une certaine mesure, on peut penser que ce réseau fonctionne à la façon d'un tamis arrêtant les particules dont le diamètre est supérieur à celui de la maille. Par exemple, il a été démontré que chez les larves de moustiques des particules de $20 \mathrm{~mm} \mu$ sont arrêtées. Chez Periplaneta, le diamètre des mailles est de 0,15 à $0,2 \mathrm{m \mu}$. Or, l'embryon hexacanthe d'Hymenolepis nana mesure environ $20 \mathrm{m \mu}$ de diamètre. Bien qu'il soit susceptible de se déformer et lde s'allonger, il lui est peut-être difficile de passer à travers un tel filtre. 


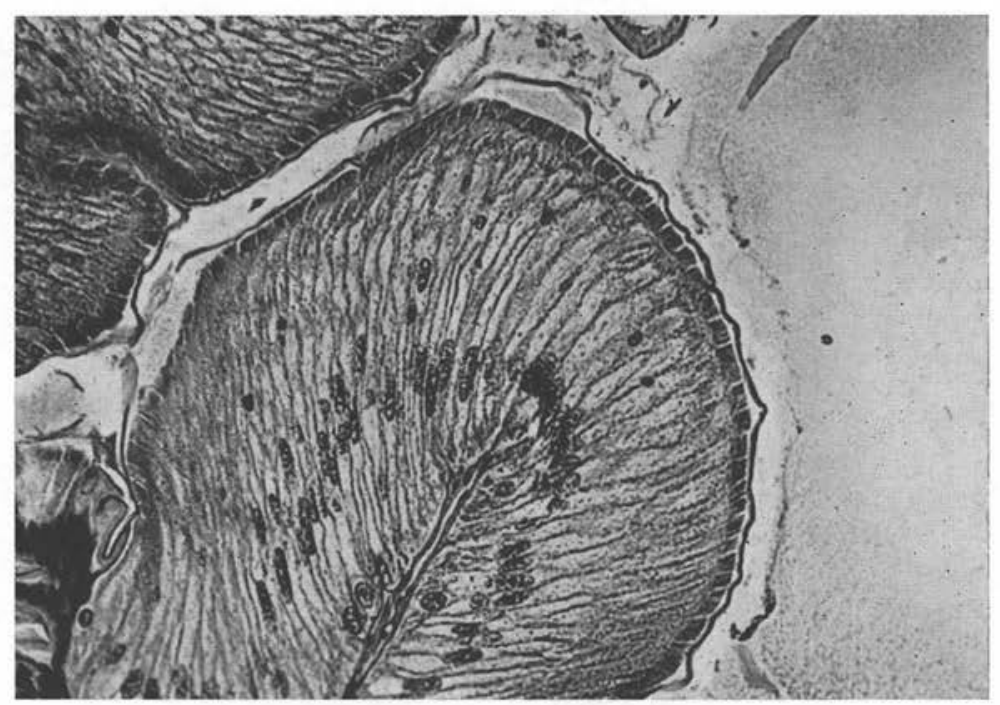

Pното 1. - Coupe d'intestin moyen de Leucophaea maderae

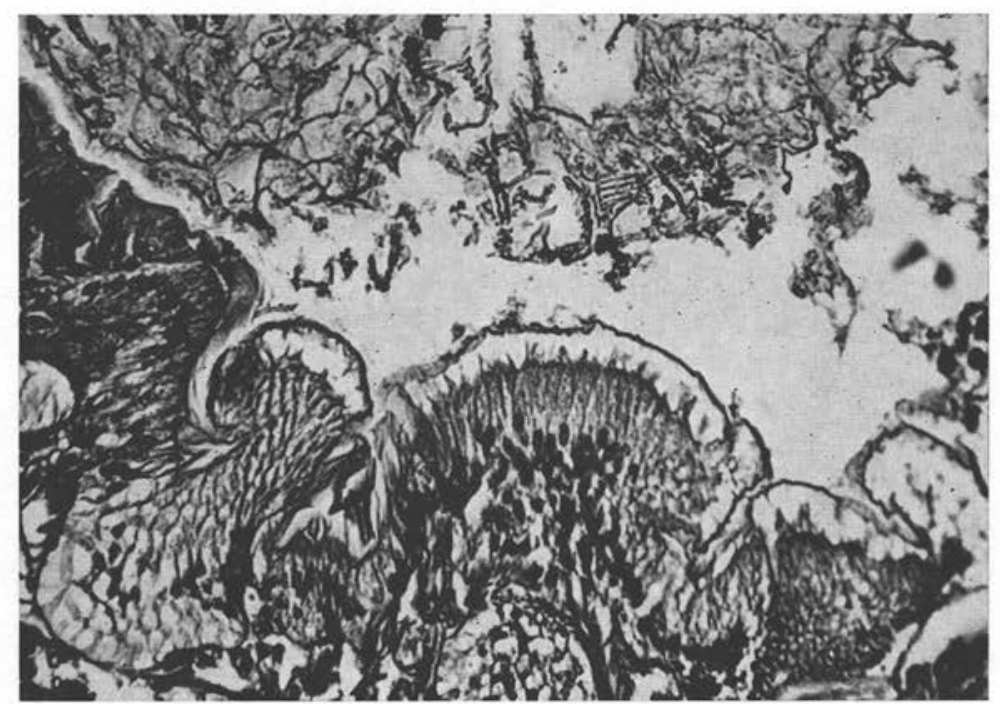

Pното 2. - Coupe d'intestin moyen de Periplaneta americana 


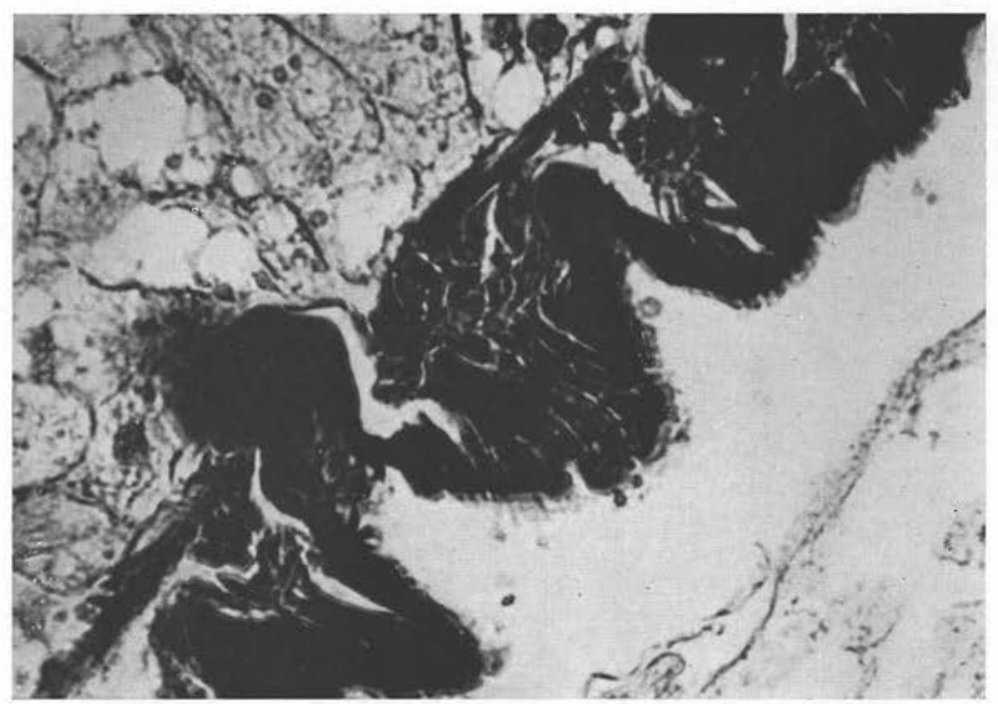

Рното 3. - Coupe d'intestin moyen de Tenebrio molitor

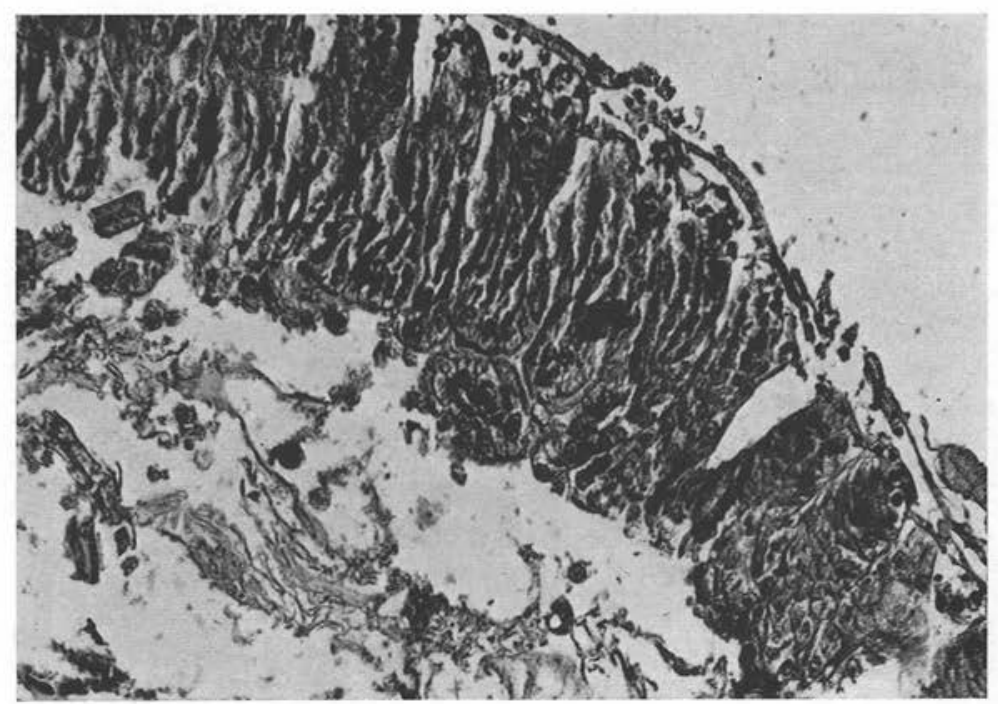

Pното 4. - Coupe d'intestin moyen de Locusta migratoria 


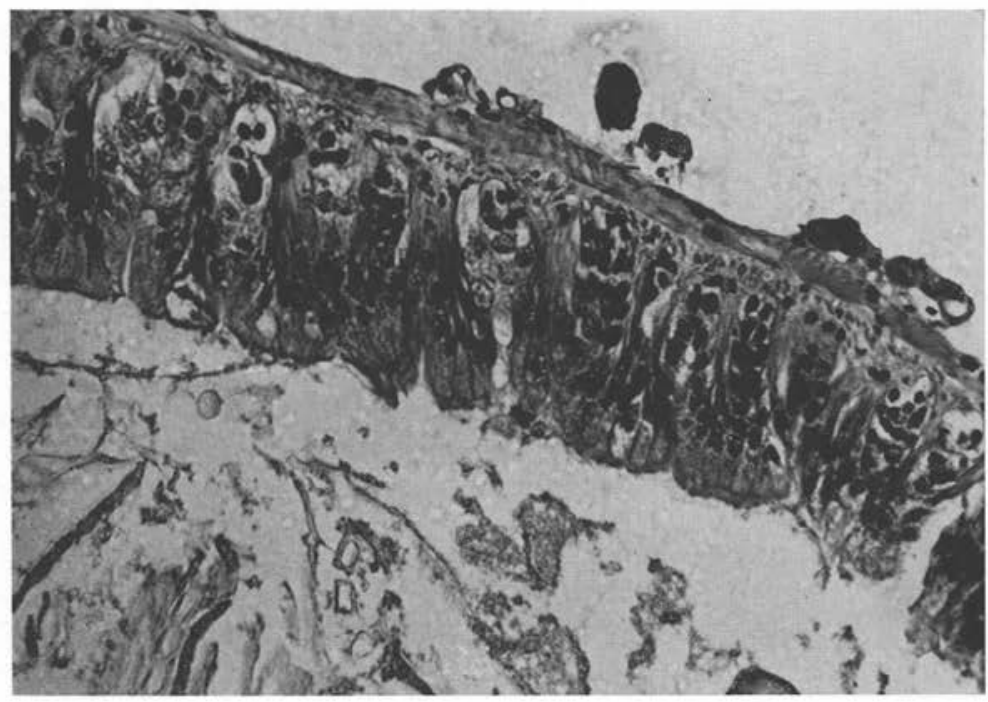

Рното 5. - Coupe d'intestin moyen de Gryllus domesticus

\section{Conclusion}

Le cysticercoïde d'Hymenolepis nana semble pouvoir s'adapter à de nombreux Insectes.

C'est la structure de la paroi intestinale et particulièrement celle de la péritrophique qui conditionne sans doute pour une espèce donnée la possibilité de s'infester.

\section{Bibliographie}

1. Cavier (R.), Léger (N.), 1965. - A propos de l'évolution d'Hymenolepis nana var. fraterna chez des hôtes intermédiaires inhabituels. Ann. Parasit. hum. et comp., $40 \mathrm{n}^{\circ}$ 6, 651-658.

2. Pujatti (D.), 1852. - Il Gryllodes sigillatus un ospite intermedio della Hymenolepis nana, Siebold. Annali del Museo civico Di storia naturale Giacomo Doria, $1947-49,63,235-241$.

3 Wigglesworth (V. B.), 1965. - In «the principles of Insect Physiology 》 Methen and Co. Ed., London. 